\title{
Is task switching nothing but cue priming? Evidence from ERPs
}

\author{
KERSTIN JosT \\ Philipps University Marburg, Marburg, Germany \\ ULRICH MAYR \\ University of Oregon, Eugene, Oregon \\ AND \\ FRANK RöSLER \\ Philipps University Marburg, Marburg, Germany
}

\begin{abstract}
Recent findings suggesting that switch costs in the task-cuing paradigm are largely attributable to a change in the task-indicating cue have been interpreted in terms of a priming model of task-switch costs (Logan \& Bundesen, 2003). According to this explanation, participants do not actually switch task sets, but merely use a cue-stimulus compound to disambiguate competing response tendencies associated with bivalent stimuli. Here, we report an event-related potential (ERP) experiment that provides evidence against this notion. In a paradigm with a 2:1 mapping between cues and tasks, we show that cue-switch and task-switch effects are dissociable on a neurophysiological level, indicating that task switching is more than a switch in the task-indicating cue. Moreover, a systematic analysis of the ERPs during the cue-stimulus interval suggests that updating processes can run in advance, before the stimulus is presented.
\end{abstract}

The task-switching paradigm has become an important tool for study of the cognitive control processes needed to flexibly adjust to a changing environment (for a review, see Monsell, 2003). In a typical task-switching experiment, participants have to switch between two or more tasks, and differences in response times (RTs) and accuracy between task alternations and task repetitions - that is, the so-called switch costs - are typically interpreted as a reflection of the processing demands involved in changing task-specific cognitive configurations. However, recent evidence has posed a challenge to this interpretation of task-switch costs, as assessed in one variant of the taskswitching paradigm - that is, the task-cuing paradigm.

In the task-cuing paradigm, tasks vary randomly from trial to trial, and a cue presented in advance indicates the relevant task. In contrast to alternative task-switching variants (e.g., that of Rogers \& Monsell, 1995), the task-cuing paradigm allows precise temporal control over both cueassociated preparatory processes and stimulus-associated processes involved in resolving stimulus-triggered competition. However, as noted independently by Logan and Bundesen (2003) and Mayr and Kliegl (2003), in the standard task-cuing paradigm, a task switch is always confounded with a switch of the task-indicating cue: Whenever the task switches, the cue switches as well; whenever the task stays the same, the cue also stays the same. To handle this problem, both groups introduced a new variant in which two cues were mapped to each task, allowing for examinations of task switching independently from cue switching. From such 2:1 mappings, three different transition types result: regular no-switch transitions (cue and task repetition); regular task-switch transitions (cue and task switch); and a new type of transition, in which cue changes are associated with task repetitions (cue-switch condition). Using this method, both studies reported substantial costs for cue switching at short cue-stimulus intervals (CSIs), even when the task remained constant. The studies, however, differed in their findings concerning the actual task-switch costs. Whereas the data in the Logan and Bundesen (2003) study suggested that task-switch costs are almost completely attributable to a cue switch, Mayr and Kliegl (2003) observed actual task-switch costs over and above the costs from a cue switch. These discrepant findings resulted in two different theoretical approaches to account for task-switch costs in the cuing paradigm.

Mayr and Kliegl's (2003) account starts with the simple assumption that working memory can contain only one coherent task set at a time, so that each task-switching trial requires updating working memory and the attentional configuration to reflect the current task demands. This updating process comes in two stages (for similar twoprocess approaches, see Meiran, 1996; Rogers \& Monsell, 
1995). The first stage, indicated by the cue-switch costs, is thought to be associated with cue-driven retrieval of task rules from long-term memory into working memory, resulting in a relatively abstract description of what has to be done with the next stimulus. In the second stage, indicated by the pure task-switch costs, the active task rules are transformed into a task-appropriate attentional configuration. In many cases, this occurs after the actual stimulus appears. However, recent evidence has suggested that under certain conditions, this attentional configuration stage can occur at least to some degree in a preparatory manner (Monsell \& Mizon, 2006).

In contrast, Logan and colleagues have proposed in successive articles a model that does not assume a genuine taskset (re)configuration process in the cued task-switching paradigm (Logan \& Bundesen, 2003, 2004; Schneider $\&$ Logan, 2005). From their initial observation of large cue-switch costs and hardly any actual task-switch costs (Logan \& Bundesen, 2003), they concluded that switch costs in the cuing paradigm do not measure endogenous task-set reconfiguration, but rather an encoding benefit when the cue from the previous trial is repeated. According to their model, cues and stimuli form compound cues, which allow for retrieving the unique response associated with each cue-stimulus combination. Cue-switch costs arise because cue repetition facilitates cue encoding. Taskswitch costs can arise because cues associated with the same task prime each other. Thus, the critical aspects here are that (1) task boundaries do not really matter, because switching occurs only between cue-stimulus compounds, and (2) task-switch and cue-switch processes represent a continuum of associative priming processes, with no clear qualitative dissociation. Thus, this priming account explicitly denies (executive) control processes in the cuing paradigm and therefore challenges those theories that attribute components of task-switch costs to task-set reconfiguration processes (e.g., Mayr \& Kliegl, 2003; Rogers \& Monsell, 1995).

It is difficult to resolve this issue with purely behavioral measures (but see Mayr, 2006; Monsell \& Mizon, 2006). The size of cue-switch or task-switch costs alone is of little help in drawing inferences about the similarities or differences between the underlying processes. However, indicators of neural activity may be helpful in this regard. In the present study, we use the high temporal resolution of the electroencephalogram (EEG) to investigate electrophysiological correlates of cue switching and task switching. For our central issue, we will examine the temporal and spatial separability of cue-switch and task-switch costs. The view that the two types of switch costs represent two distinct sets of processes implies that they should be distinct on the neural level, both in timing and in terms of neuroanatomical localization. In contrast, the priming account predicts no clear temporal or spatial separation between the neurallevel effects associated with the cue-switch versus the taskswitch cost. To decide between these two alternatives, we recorded event-related brain potentials (ERPs) for different transition types in a task-cuing paradigm.

On the basis of their behavioral results, Mayr and Kliegl (2003) proposed that cue-driven retrieval of task rules can occur prior to the arrival of the next stimulus, whereas attentional reconfiguration needs to wait until stimulus presentation. This view has been recently challenged by behavioral findings suggesting that, under certain conditions, pure task-switch costs can be reduced with increasing intervals between the cue and the stimulus (Monsell \& Mizon, 2006). The EEG provides the opportunity to directly measure what happens within the CSI. When using cued task-switching paradigms, a consistently reported finding is that the cue elicits a relative positivity for switch as compared with repetition trials (see, e.g., Nicholson, Karayanidis, Bumak, Poboka, \& Michie, 2006; Nicholson, Karayanidis, Poboka, Heathcote, \& Michie, 2005; Rushworth, Passingham, \& Nobre, 2002, 2005). Some authors have directly related this positive deflection to the $\mathrm{P} 3 \mathrm{com}$ ponent of the ERP (see, e.g., Barceló, Escera, Corral, \& Periáñez, 2006; Barceló, Periáñez, \& Knight, 2002). The P3 is assumed to reflect endogenous or cognitive aspects of "context updating"- that is, the comparison of the attributes of incoming stimuli with an internal model and the subsequent revision of the model (Donchin, 1981; see also Donchin \& Coles, 1988). Accordingly, in task switching the larger P3 for switch than for repetition trials has been interpreted as reflecting updating of task sets in working memory (e.g., Barceló et al., 2002). So far, the degree to which the P3 increase actually responds to a change in task, versus only to a change in cue, is unknown (but see Nicholson et al., 2006). From the results of Monsell and Mizon (2006), we might expect not only cue-switch (relative to cue-repetition) transitions, but also task-switch (relative to cue-switch) transitions, to lead to an increased P3.

According to Mayr and Kliegl (2000), long-term memory retrieval of task rules can be triggered by any signal that indicates an upcoming task. An updating operation, therefore, might be elicited on all trials, even when the task and the cue are repeated. In contrast, trial-by-trial updating is not necessary in situations in which the taskrelevant rules do not change across successive trials - that is, in single-task blocks. Thus, to experimentally isolate the updating operation, we need to compare no-switch trials from mixed-task blocks with those from single-task blocks (see also Kray, Eppinger, \& Mecklinger, 2005). From the working memory updating view, we expect to find a P3 component for the cue even on no-switch trials from mixed blocks, but no comparable effect for the cue on single-task blocks. To further substantiate the updating view, we will also make an attempt to relate $\mathrm{P} 3$ amplitude to performance. Specifically, we expect that more efficient updating (i.e., larger P3 amplitudes) should result in faster performance.

We used a variant of the basic paradigm introduced by Mayr and Kliegl (2003): Participants switched between color and shape discriminations, and each of the tasks could be signaled by two arbitrary letter cues, allowing us to compare no-switch, cue-switch, and task-switch transitions. The CSI was set to either 200 or $1,000 \mathrm{msec}$. This manipulation permitted us to compute the reduction of switch costs given the opportunity to prepare for the upcoming task. Moreover, with the CSI 1,000 condition, we could measure the electrophysiological correlates of task-set preparation. 
In addition to the task-switching situation, we also used a blocked, single-task condition, which otherwise had the same temporal characteristics as the task-switching condition. This condition was included in order to obtain information about the baseline ERP responses to the cue and the stimulus when no task switching was required.

\section{METHOD}

\section{Participants}

A total of 16 right-handed students from the University of Marburg ( 14 women, 2 men; mean age $=22$ years) participated. All were native speakers of German, had normal or corrected-to-normal vision, and were naive with respect to the purpose of the study. They gave informed consent and received either course credit or monetary compensation.

\section{Stimuli and I'ask}

The participants had to discriminate either the shape or the color of a stimulus. The stimuli were four different shapes (circle, triangle, square, or cross) that appeared in four different colors (red, blue, green, or yellow). A letter cue presented in advance informed each participant about the relevant dimension. The letters "G" and "S" indicated the color task; "W" and "B" indicated the shape task. Responses were given with the index and middle fingers of both hands, using four keys of the computer keyboard $(X, C, N$, and $M)$ that were covered with white stickers. All other keys were covered with black stickers. The response keys (from left to right) were mapped to colors and shapes, as follows: green and circle on the first, blue and cross on the second, yellow and triangle on the third, and red and square on the fourth.

\section{Procedure}

Participants sat in a dimly lit and electrically shielded experimental chamber in front of a computer screen, which was located at eye level at a distance of about $70 \mathrm{~cm}$. All stimuli appeared centrally on a black screen within a white frame ( $3 \mathrm{~cm}$ in width, $4.5 \mathrm{~cm}$ in height) that was visible throughout the trial. A trial started with the presentation of a fixation sign for $500 \mathrm{msec}$. The task cue and stimulus (both with a height of $0.8 \mathrm{~cm}$ ) were then presented sequentially. Depending on the CSI, the cue was replaced by the stimulus after either 200 or $1,000 \mathrm{msec}$. The stimulus remained visible until the response was given, but no longer than $2,000 \mathrm{msec}$. Participants were instructed to respond as quickly and accurately as possible, and they immediately received visual feedback about errors and delayed responses (delayed responses occurred in less than $0.5 \%$ of the trials). The response-stimulus interval was $2,500 \mathrm{msec}$ in both CSI conditions.

Trials were presented in blocks of 32 trials each. The experiment comprised 24 blocks in which participants had to switch between the color and shape tasks. CSI was constant throughout a block and changed every three blocks. In addition, participants received 16 single-task blocks (four for each task $\times$ CSI combination); one half were presented before the mixed blocks, and the other half after. CSI $\times$ task combination changed every second block. All blocks were separated by short breaks, which could be terminated by a buttonpress. Stimulusresponse mappings were presented at the beginning of each block in the form of a diagram representing the four shapes (in black), the four colors (as color patches), and the corresponding response keys.

For each participant, a new trial sequence was created with the following restrictions. The three critical transition types occurred equally often in the mixed blocks $(p=.33)$; in single-task blocks, cue switches and repetitions both occurred with $p=.5$ (i.e., 128 trials of each transition type $\times$ CSI combination). The 16 colorshape combinations appeared equally often in each task, CSI, and transition type condition. Of these 16 combinations, 4 were response congruent, comprising $25 \%$ of the trials.

Following Mayr and Kliegl (2003), each participant received only one of the two possible cue transitions in case of a task switch (e.g., "S" to "W" and "G" to "B" for color-shape transitions). Prior to the experimental session, the participants were familiarized with the tasks in a practice session with six single-task blocks (three consecutive blocks for each task) and three mixed blocks.

\section{EEG Recording, Artifact Handling, and Signal Extraction}

The EEG was recorded with $61 \mathrm{Ag} / \mathrm{AgCl}$ electrodes inserted in an elastic cap (EASYCAP, Munich, Germany) with predefined electrode positions, extrapolated from the 10-20 system (Jasper, 1958). The electrodes were referenced to the nose tip. The horizontal and vertical electrooculograms were monitored with electrode pairs attached at the outer canthi of both eyes and the sub- and supraorbital ridges of one eye. The left or right mastoid served as the ground. The impedances of all electrodes were kept below $5 \mathrm{k} \Omega$. Signals were sampled with two 32-channel amplifiers (SynAmps, Neuroscan) at $500 \mathrm{~Hz}$ and filtered with a band-pass (DC to $40 \mathrm{~Hz}$ ) and a notch $(50 \mathrm{~Hz})$ filter. A DC reset was initiated prior to every second block. Drift artifacts were corrected by a regression method (Hennighausen, Heil, \& Rösler, 1993). The EEG was segmented into epochs starting $100 \mathrm{msec}$ before the onset of the cue and ending 1,000 msec after stimulus onset (i.e., 1,300-msec-long epochs for the CSI 200 condition and 2,100-msec epochs for the CSI 1,000 condition). Epochs containing eye blinks (detected by means of cross-correlations with a template) or other artifacts (detected by a threshold criterion-i.e., a voltage range within a segment of $>200 \mathrm{~V}$ ) were removed. ERPs were extracted by averaging epochs separately for participants, electrodes, and experimental conditions, and referenced to a $100-\mathrm{msec}$ baseline preceding the onset of the cue. All averages (except for the RT-based analysis; see below) were based on a minimum of 45 trials (maximum $=94$ trials, $M=75$ trials).

\section{Statistical Analysis}

The first trial of each block, trials with incorrect responses, trials following incorrect responses, and trials with response repetitions (see Mayr \& Kliegl, 2003) were excluded from the RT and ERP analyses. Trials were classified as no-switch, cue-switch, and taskswitch trials according to their relationship to the preceding trial. No-switch and cue-switch transitions in the single-task blocks were combined, because a first analysis did not reveal any differences between them for either the ERP or the behavioral data.

Following Mayr and Kliegl (2003), two contrasts were used, comparing the no-switch and cue-switch conditions (cue-switch contrast) and the cue-switch and task-switch conditions (task-switch contrast). This procedure allowed for decomposing the total switch costs into the cue-switch and task-switch components. In a third contrast, no-switch trials in the mixed blocks were compared with trials in the single-task blocks.

The statistical analysis of the ERPs was based on the same contrasts and consisted of repeated measure ANOVAs with the factors transition type and electrode, calculated separately for consecutive time windows $50 \mathrm{msec}$ in width. Nineteen standard electrodes according to the 10-20 system (see Figure 1C) were used for the statistical analysis. These were uniformly distributed over the scalp and were sufficient to capture potential topographical differences. "Local" tests (i.e., for each electrode) were calculated only for those time windows in which the superordinate ANOVA had signaled significant differences between the conditions. $F$ statistics were corrected according to Huynh and Feldt (1976). The uncorrected degrees of freedom, the corrected $p$ value, and the respective $\varepsilon$ values are reported.

\section{RESULTS}

In presenting our results, we will first report the behavioral and then the ERP data. Within each section, we will first focus on the more general issue of global updating costs (i.e., no-switch trials from mixed vs. single-task blocks) and then on the more specific question of differences between cue-switch and task-switch transitions. 


\section{Behavioral Data}

Table 1 shows RTs and error rates both for the three transition types in the mixed blocks and for the singletask blocks. An ANOVA with the factors CSI (2 levels) and transition type (4 levels) yielded main effects of CSI $[F(1,15)=78.92, p<.0001]$ and transition type $[F(3,45)=195.30, p<.0001]$, as well as a significant interaction $[F(3,45)=95.30, p<.0001]$. RTs decreased with increasing time to prepare $[F(1,15)=46.85,90.57$, and 146.11 for no switch, cue switch, and task switch, respectively; all $p \mathrm{~s}<.0001$ ], except for the single-task blocks, in which CSI had a negligible effect $(10 \mathrm{msec}$; $F<1$ ). RTs in the single-task blocks were generally slower than those in the mixed blocks. The direct comparisons of trials in single-task blocks with no-switch trials in the mixed blocks were highly significant $[F(1,15)=365.75$ and 42.16, both $p$ s $<.0001$, for the CSI 200 and CSI 1,000 conditions, respectively]. So far, the data showed the typical pattern of mixing costs and preparation.

Within the mixed blocks, RTs were generally slower for the task-switch than for the no-switch condition. With a short preparatory interval, a part of the total switch costs was associated with the cue switch $(23 \mathrm{msec}, S E=$ $7.41)$ and another component with the actual task switch $(118 \mathrm{msec}, S E=9.91)$. Both the cue-switch contrast $[F(1,15)=9.26, p=.0082]$ and the task-switch contrast $[F(1,15)=141.22, p<.0001]$ proved to be reliable. However, when the preparatory interval was long, the cue-switch costs were nearly eliminated (4 msec, $S E=$ $8.18 ; F<1)$, whereas "true" task-switch costs of $30 \mathrm{msec}$ $[S E=8.88 ; F(1,15)=11.28, p=.0043]$ remained. These were, however, significantly reduced in comparison with the costs in the CSI 200 condition $[F(1,15)=112.91, p<$ .0001 , for a direct comparison of task-switch costs in the CSI 1,000 and CSI 200 conditions].

Error rates showed the same trend. An ANOVA yielded main effects of CSI $[F(1,15)=11.17, p=.0045]$ and transition type $[F(3,45)=21.35, p<.0001]$, as well as a marginally significant interaction $[F(3,45)=2.47, p=$ .0743]. Participants committed significantly more errors in the CSI 200 than in the CSI 1,000 condition. This effect was not significant in the single-task blocks. Cue-switch and task-switch costs were also obvious in the error rates. However, only the difference between cue switch and task switch was significant $[F(1,15)=13.94, p=.0020$, and $F(1,15)=17.83, p=.0007$, for the CSI 200 and CSI 1,000 conditions, respectively].

\section{ERPs}

Mixed versus single-task blocks. Figure 1 shows the comparison of ERPs evoked in the mixed and single-task blocks separately for the two CSI conditions. Mixed-block trials in the CSI 1,000 condition (Figure 1B) show two positive deflections with maximal amplitude over parietal areas, one elicited by the cue and the other by the stimulus. In single-task blocks, in which the cue is not relevant for task performance, only the stimulus evoked a pronounced positive deflection. Comparable differences between single-task and mixed blocks are also observable in the CSI 200 condition (see Figure 1A), although cue- and stimulus-related potentials are superimposed because of the short interval between cue and stimulus. There, a sustained positive deflection with two peaks between 300 and $800 \mathrm{msec}$ emerged in the mixed blocks (see electrode Pz), whereas single-task blocks elicited a much later positivity with only one peak.

In the CSI 1,000 condition, between 350 and $650 \mathrm{msec}$ the cue-evoked positivity for mixed-block trials (noswitch condition) significantly differed from the ERPs in single-task blocks $[\min F(1,15)=8.35, p=.0112$; $\max F(1,15)=36.99, p<.0001$; the terms $\min F$ and $\max F$ refer to the smallest and largest $F$ values in the reported time interval]. This effect interacted with electrode during the same time windows $[\min F(18,270)=3.10, p=.0216$, $\varepsilon=.2238 ; \max F(18,270)=24.89, p<.0001, \varepsilon=.2900]$. The effect was maximal at parietal sites (Pz and surrounding electrodes), but reached significance at all electrodes $(p<.05)$. During stimulus processing, the amplitude of the positivity was larger overall in the single-task blocks and was significantly different from the no-switch condition between 1,400 and 1,600 msec at posterior electrodes $[\mathrm{Cz}, \mathrm{P} 3, \mathrm{Pz}, \mathrm{P} 4, \mathrm{~T} 5, \mathrm{~T} 6, \mathrm{O} 1$, and $\mathrm{O} 2$; transition type $\times$ electrode interaction: $\min F(18,270)=9.62, p<.0001$, $\varepsilon=.2519 ; \max F(18,270)=12.65, p<.0001, \varepsilon=.2707]$, with the maximum effect at Pz. In the CSI 200 condition, differences between single-task and mixed blocks were significant between 250 and $550 \mathrm{msec}[\min F(1,15)=$ $8.68, p=.0100 ; \max F(1,15)=93.28, p<.0001]$ and again between 700 and $850 \mathrm{msec}[\min F(1,15)=3.48$, $p=.0818 ; \max F(1,15)=10.85, p=.0049]$.

To summarize this section, comparisons between the mixed and single-task blocks revealed differences for cue processing. Task-relevant cues triggered a positive deflection that was absent in single-task blocks. This is consistent with the notion that cues initiate an updating process that can occur in a preparatory manner, prior to stimulus arrival.

Table 1

Mean Response Times (RTs, in Milliseconds) and Error Rates

for Single-Task Blocks and for the Three Transition Types

in Mixed Blocks, Separately for the Short and Long Cue-Stimulus Intervals (CSIs)

\begin{tabular}{|c|c|c|c|c|c|c|c|c|}
\hline \multirow[b]{3}{*}{ Transition } & \multicolumn{4}{|c|}{ CSI $200 \mathrm{msec}$} & \multicolumn{4}{|c|}{ CSI $1,000 \mathrm{msec}$} \\
\hline & \multicolumn{2}{|c|}{ RT } & \multicolumn{2}{|c|}{$\%$ Error } & \multicolumn{2}{|c|}{ RT } & \multicolumn{2}{|c|}{$\%$ Error } \\
\hline & $M$ & $S E$ & $M$ & $S E$ & $M$ & $S E$ & $M$ & $S E$ \\
\hline Single task & 708 & 21 & 2.79 & 0.59 & 698 & 23 & 2.28 & 0.35 \\
\hline No switch & 906 & 22 & 5.77 & 1.09 & 779 & 25 & 3.75 & 0.90 \\
\hline Cue switch & 929 & 23 & 6.31 & 1.11 & 783 & 21 & 4.64 & 0.87 \\
\hline Task switch & 1,047 & 24 & 12.57 & 2.34 & 813 & 23 & 9.09 & 1.52 \\
\hline
\end{tabular}



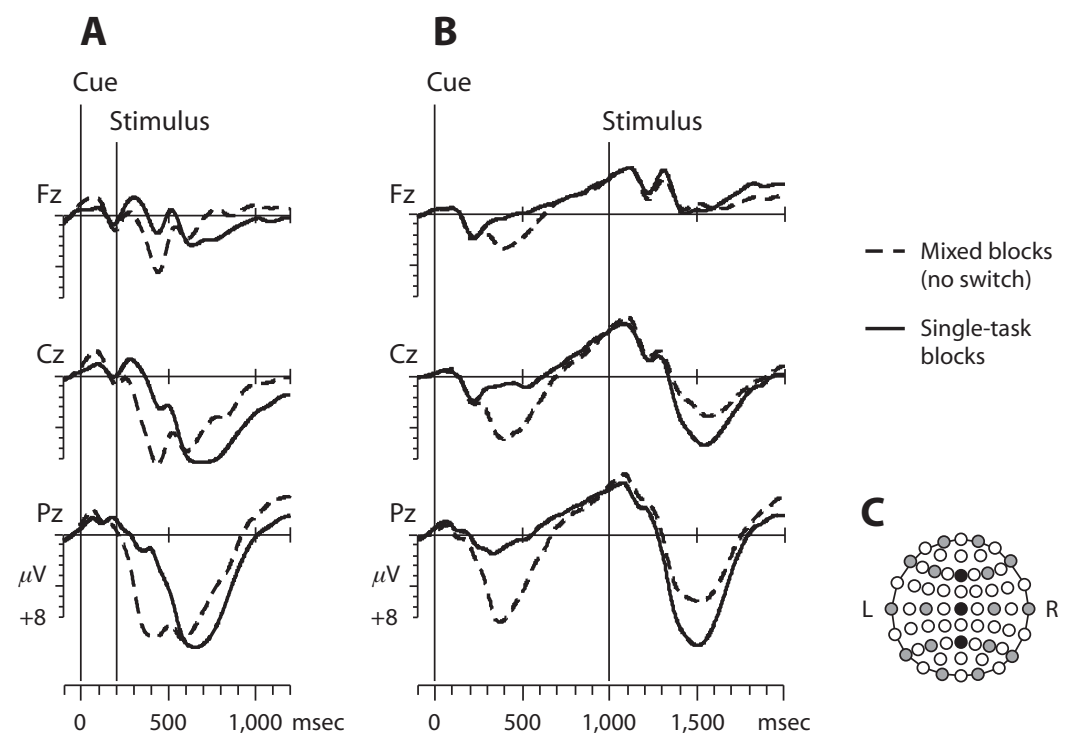

Figure 1. (A, B) Grand average ERPs $(n=16)$ of no-switch trials in the mixed blocks and of trials in the single-task blocks, measured at three midline electrodes. In this figure and the following ones, all ERPs are referenced to a 100-msec baseline preceding the onset of the cue, and negativity is plotted upward. Different ERP shapes are indicated for short and long cue-stimulus intervals (CSIs). In the CSI 1,000 condition (B), the cue and the stimulus evoked separate ERP components, whereas in the CSI 200 condition (A), these components overlapped. In mixed blocks with the long CSI, both the cue and the stimulus evoked positive deflections with maximal amplitude over parietal areas. In single-task blocks, in which the cue was not relevant for task performance, only the stimulus evoked a pronounced positive deflection. A comparable difference is also observable in the CSI 200 condition. There, a sustained positive deflection with two peaks between 300 and 800 msec emerged in the mixed blocks, whereas single-task blocks elicited a much later positivity with only one peak. (C) Electrode positions of the 19 electrodes included in the statistical analyses. The electrodes for which voltage-time plots are shown $(\mathrm{Fz}, \mathrm{Cz}$, and $\mathrm{Pz})$ are marked in black.

Task-switch versus cue-switch transitions. Figure 2 shows the ERPs for the three transition types in the mixed blocks separately for the short- and long-CSI conditions. Here again, the different wave shapes are apparent, with cue-evoked and stimulus-evoked parietal positivities for the CSI 1,000 condition and an overlap of the ERPs in the CSI 200 condition. Despite the different ERP time courses, the two CSI conditions showed similar effects with respect to the differences between the switching conditions. In both CSI conditions, cue-switch and taskswitch trials evoked a more negative potential than did no-switch trials over frontal electrodes between 300 and $400 \mathrm{msec}$ after cue onset. At around 400 to $600 \mathrm{msec}$, differences between transition types are also obvious in the parietal positivity. These differences in the P 3 can only be interpreted for the CSI 1,000 condition, because here cue-evoked ERPs do not overlap with the stimulus-evoked ERPs. These results will be reported below, following the effects that are similar for both CSI conditions.

As can be seen in Figure 3, showing the difference waves of the critical contrasts, the early cue-switch effect with a frontal maximum is separable from a later task-switch effect: Task switching evoked a relative negativity when compared with both cue switching and no switching. This difference was present a few hundred milliseconds after stimulus presentation (i.e., around $1,500 \mathrm{msec}$ after cue onset in the CSI 1,000 condition and between $800 \mathrm{msec}$ and the end of the trial when CSI was short) and was broadly distributed across electrodes. Thus, the total difference between a task switch and no switch can be separated into two components, an early one attributable to a cue switch and a late one that is due to the actual task switch.

Consistent with these observations, both the cue-switch and task-switch contrasts proved to be highly reliable, and they reached significance in nonoverlapping time windows. The ANOVAs contrasting cue switches and no switch in the CSI 200 condition (i.e., the cue-switch contrast) revealed significant main effects of transition type between 300 and $400 \operatorname{msec}[\min F(1,15)=8.14, p=$ $.0121 ; \max F(1,15)=11.52, p=.0040]$ and significant interactions of transition type $\times$ electrode between 300 and $450 \mathrm{msec}[\min F(18,270)=6.06, p=.0003, \varepsilon=$ $.2324 ; \max F(18,270)=6.50, p<.0001, \varepsilon=.2662]$. For the CSI 1,000 condition, the relative negativity for cue switches as compared with no switch was smaller, but nevertheless it yielded a marginally significant effect between 300 and $400 \mathrm{msec}[\min F(1,15)=3.10 p=.0985$; $\max F(1,15)=3.30, p=.0891]$ that interacted significantly with electrode in the time window between 350 and $400 \mathrm{msec}[F(18,270)=2.92, p=.0464, \varepsilon=.1612]$. 

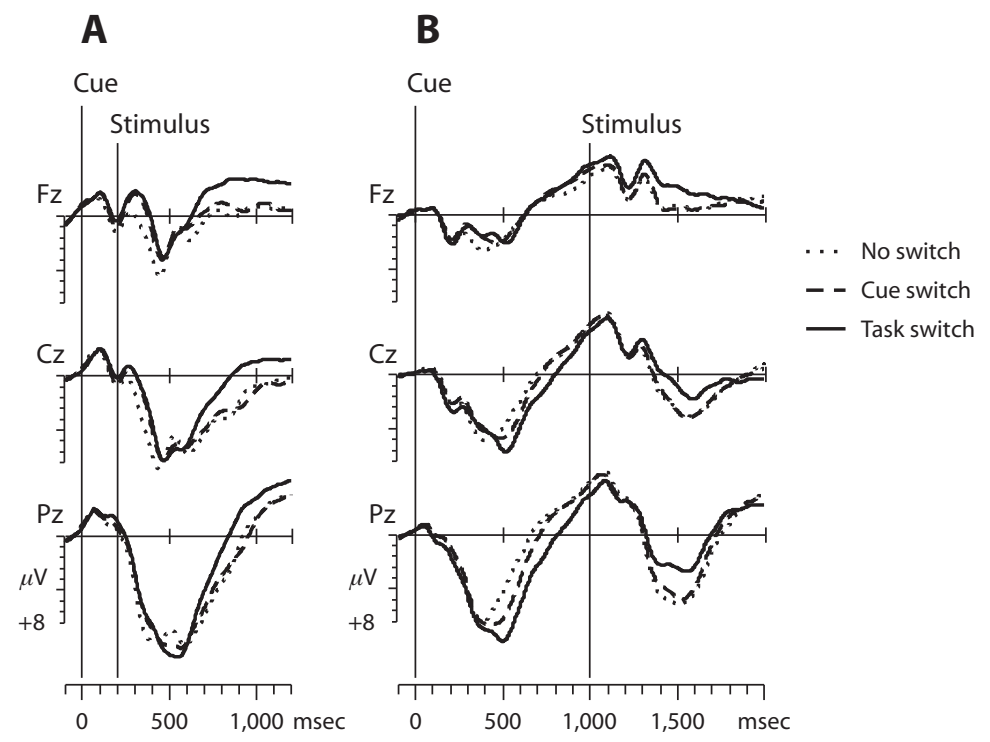

Figure 2. Grand average ERPs of no-switch, cue-switch, and task-switch trials. During cue processing in the CSI 1,000 condition (B), differences had already emerged after 300 msec. There, cue-switch and task-switch trials evoked more negative potentials than did no-switch trials at frontal electrodes (see Fz). This difference was followed by an effect in the parietal positivity that extended into the contingent negative variation. The amplitude of the positivity was larger for task-switch than for no-switch trials, and cue-switch ERPs lay in-between. After stimulus onset, the curves completely converge between 1,100 and 1,200 msec (without showing significant differences), before emerging into another positive deflection. During the latter interval, task-switch trials elicited more negative potentials than did cue-switch and no-switch trials. Both negative differences - that is, the early cue-switch and the later task-switch effectare also evident with the short CSI (A).

The task-switch contrast was significant between 650 and $1,200 \mathrm{msec}$ in the CSI 200 condition $[\min F(1,15)=4.87$, $p=.0434 ; \max F(1,15)=21.00, p=.0004]$ and interacted with electrode between 800 and $950 \mathrm{msec}[\min F(18,270)=$ $2.09, p=.0987, \varepsilon=.2089 ; \max F(18,270)=3.61, p=$ $.0084, \varepsilon=.2431]$. In the CSI 1,000 condition, the difference between task and cue switches was significant between 1,400 and $1,600 \mathrm{msec}[\min F(1,15)=3.50, p=$ $.0812 ; \max F(1,15)=6.55, p=.0218]$.

The cue-switch and task-switch contrasts differed not only with respect to their temporal characteristics, but also with respect to their topographical distributions (see Figure 3B). For both CSI conditions, the maximum of the cue-switch-related negative difference was focused around frontocentral electrodes $(\mathrm{FCz}$ and the surrounding electrodes), whereas the task-switch-related negative difference was more broadly distributed. The cue-switch contrast for the short preparatory interval was significant at all frontal, central, and parietal electrodes $(p<.05$ between 300 and $400 \mathrm{msec}$, except at electrode $\mathrm{Pz}$, which was significant with $p<.05$ between 300 and $350 \mathrm{msec}$ only; see Figure $3 \mathrm{~A}$ ). In the CSI 1,000 condition, the effect reached significance at $\mathrm{Fz}$ and $\mathrm{Cz}$ between 300 and $400 \mathrm{msec}$, and reached marginal significance $(p<.07)$ at the surrounding electrodes F3, F4, and C4. The larger focus on frontal areas is further substantiated by a direct comparison of frontal (F3, Fz, and F4) and parietal (P3, Pz, and P4) electrodes, which reached significance $(p<.05)$ in the CSI 200 condition and was marginally significant $(p<.1)$ in the CSI 1,000 condition. In contrast, the task-switch contrasts did not show any significant differences between frontal and parietal electrodes. Moreover, between 800 and $900 \mathrm{msec}$, the effect reached significance at all electrodes in the CSI 200 condition and at almost all medial electrodes (F3, Fz, $\mathrm{C} 3, \mathrm{Cz}, \mathrm{P} 3, \mathrm{Pz}$, and P4), as well as at F7 and at the temporal electrodes T3 and T4, in the CSI 1,000 condition. To further substantiate these topographic differences between the cue-switch and task-switch contrasts, an ANOVA with the factors contrast and CSI was run with $z$-standardized difference scores (see McCarthy \& Wood, 1985). This analysis revealed a significant interaction of contrast $X$ electrode $[F(18,270)=2.82, p=.0214, \varepsilon=.2055]$ and no effects of CSI, indicating that the topographies differed for the types of contrasts, but not for the CSI conditions.

To conclude, the cue-switch and task-switch contrasts were dissociable in both time and topography, with remarkably similar temporal order and topographical distributions of the effects across the CSI conditions. This indicates that cue switching and task switching can be attributed to two distinct processes, one associated with the encoding or interpretation of the cue and the other with task performance.

P3 during the preparatory interval. As stated above, the cue in the CSI 1,000 condition elicited a pronounced positive deflection between 200 and $700 \mathrm{msec}$ that was not pres- 


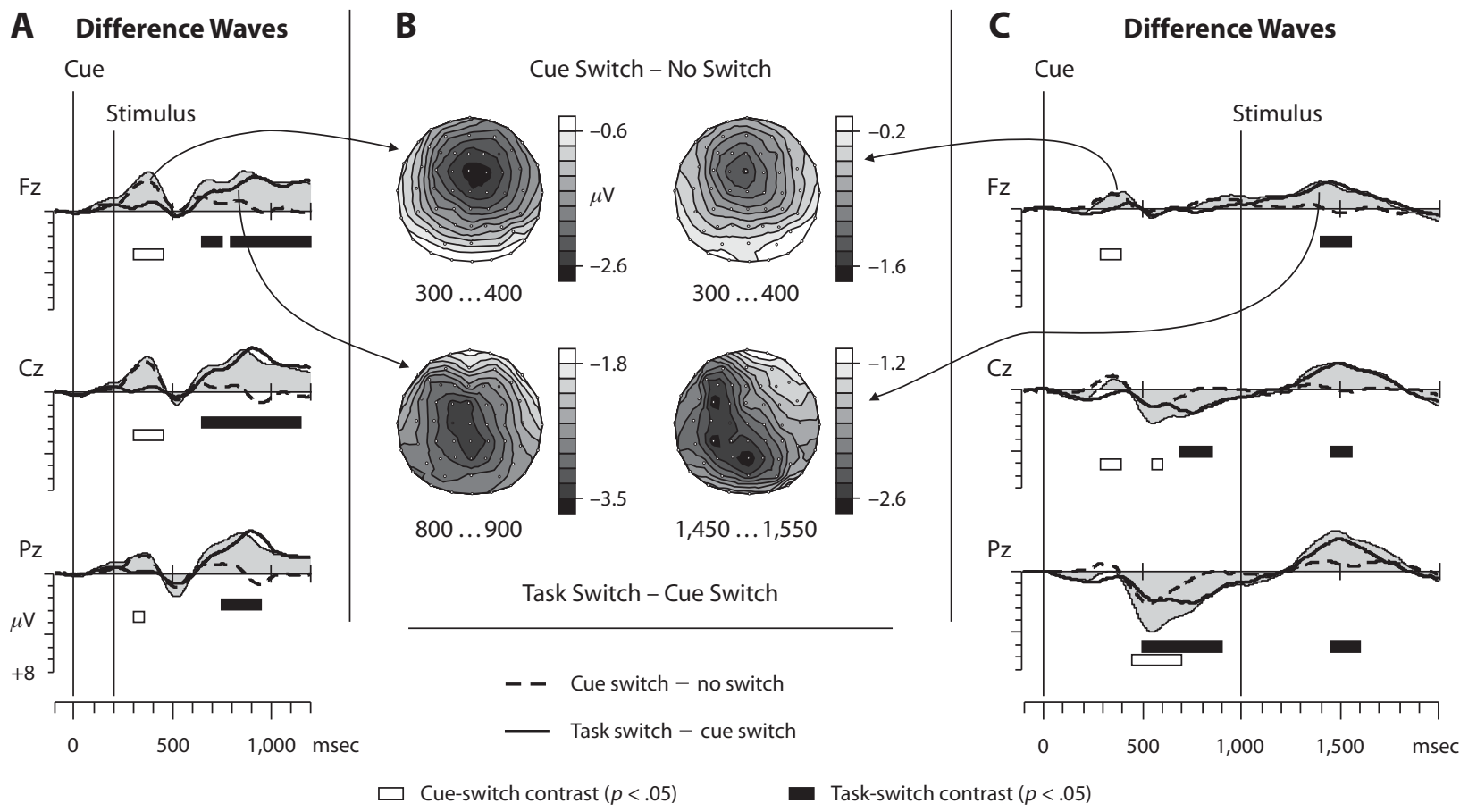

Figure 3. Difference waves for the CSI 200 (A) and CSI 1,000 (C) conditions. The shaded area represents the ERP difference for task-switch minus no-switch trials. For both CSI conditions, this "total" switch effect is reflected by two negative differences that emerge in separate time windows - that is, an early negative difference between 300 and 400 msec and a later one starting a few hundred milliseconds after stimulus onset. In addition, a large positive difference is present in the CSI 1,000 condition, which reflects the differences in the cue-triggered positivity (see Figure 2). The dashed and solid lines depict the cue-switch contrast (cue switch minus no switch) and the task-switch contrast (task switch minus cue switch), respectively. These graphs show that the early part of the total switch effect is due to the cue switch, whereas the late part is due to the actual task switch. Horizontal bars indicate the time windows of significant differences. In panel B, the distinct topographies of the cue-switch and task-switch contrasts appear. The maps, derived from all 61 electrodes, show the time windows in which the effects were maximal.

ent in single-task blocks, thus indicating that the positivity reflects cue-triggered preparation. Latency and topography qualify this as a $\mathrm{P} 3$ component (more precisely, as the $\mathrm{P} 3 \mathrm{~b}$ subcomponent). As is evident from Figure $2 \mathrm{~B}$, during the epoch of maximum amplitude, the positivity was systemically affected by the necessity to switch. The amplitude was larger for task-switch than for cue-switch and no-switch trials, and this difference extended into the contingent negative variation. Similar switch-related positive effects have been described in previous studies and interpreted as reflecting advance reconfiguration (see, e.g., Nicholson et al., 2006).

The differences between the transition types were substantiated by the ANOVAs: The cue-switch contrast was marginally significant between 500 and $600 \mathrm{msec}$ $[\min F(1,15)=3.78, p=.0708 ; \max F(1,15)=4.34, p=$ $.0548]$ and interacted with electrode between 450 and $700 \mathrm{msec}[\min F(18,270)=2.88, p=.0257, \varepsilon=.2433$; $\max F(18,270)=5.92, p=.0011, \varepsilon=.1838]$. Electrodewise comparisons showed that the amplitude of the positivity was significantly larger for cue-switch than for cuerepetition trials at parietal $(\mathrm{P} 3, \mathrm{P} 4$, and $\mathrm{Pz}$ between 450 and $700 \mathrm{msec})$ and central $(\mathrm{C} 3, \mathrm{Cz}$, and $\mathrm{C} 4$ between 500 and $600 \mathrm{msec}$ ) electrodes.

The contrast between task-switch and cue-switch transitions reached significance between 700 and $850 \mathrm{msec}$ $[\min F(1,15)=4.95, p=.0418 ; \max F(1,15)=8.53, p=$
$.0105]$ and interacted with electrode between 450 and $900 \mathrm{msec}[\min F(18,270)=4.59, p=.0028, \varepsilon=.2197$; $\max F(18,270)=10.73, p<.0001, \varepsilon=.2846]$. The effect was more broadly distributed than the cue-switch contrast and reached significance at parietal $(\mathrm{Pz}, \mathrm{P} 3$, and $\mathrm{P} 4 \mathrm{be}-$ tween 500 and $850 \mathrm{msec})$ and central $(\mathrm{C} 3, \mathrm{C} 4$, and $\mathrm{Cz}$ between 700 and $850 \mathrm{msec}$ ) electrodes, extending to temporal and occipital sites (T5, T6, O1, and O2, significant between 500 and $850 \mathrm{msec}$ ). Again, the maximum effect size was found at $\mathrm{Pz}$.

A close inspection of Figure $2 \mathrm{~B}$ reveals that the ERP differences within the $\mathrm{P} 3$ time window are caused by both amplitude and latency variations. In order to disentangle amplitude and latency differences, we identified the time point of the maximum amplitude for each participant and transition type at electrode Pz. For this analysis, the ERPs were smoothed with a 7-Hz low-pass filter. We searched for the P3 in the time window between 200 and $700 \mathrm{msec}$. In all cases, a positive component could be identified, and in all cases the peak maximum was found between 300 and $600 \mathrm{msec}$ (with 322 and $554 \mathrm{msec}$, respectively, for the smallest and largest latencies). The cue-switch contrast was associated with a significant latency shift of $50 \mathrm{msec}$ $[F(1,15)=10.21, p=.0060]$, and another significant delay of $32 \mathrm{msec}$ was due to a task switch $[F(1,15)=$ $4.92, p=.0423]$. In contrast, a significant increase in 
peak amplitude was observed only for actual task switches $[F(1,15)=7.79, p=.0137]$. This result pattern is compatible with the idea that the P3 amplitude reflects the amount of context updating (Donchin \& Coles, 1988); here, it suggests that more context updating is needed when a switch in task occurs than when the task stays the same.

To further validate that the $\mathrm{P} 3$ is related to preparation, it must be shown that its amplitude covaries with task performance, assuming that efficient updating leads to increased performance. We therefore investigated whether the P3 amplitude covaried with trial-by-trial variations in response speed. This idea is based on the observation by De Jong (2000) that trial-by-trial variations in response speed are due to differences in preparation during the CSI. The fastest trials of the RT distribution should be those that are successfully prepared, and this should be indicated by a larger P3 during the preparatory interval. To test this prediction, we sorted the trials of each participant by RT, split the data into quintiles, and averaged the EEGs for the resultant bins. We decided to use quintiles because this allowed for averaging a sufficient number of trials in each bin (between 30 and 52 trials, $M=44$ trials), with RT differences between the highest and lowest bins reaching a sufficient size $(M=624 \mathrm{msec})$ to allow for the assumption that the effectiveness of preparation/updating was different across bins. In order to control for RT differences between switch and repetition trials, this procedure was applied separately to the three transition types, which were aggregated afterward. One of the participants had to be excluded because of too few trials. The cuelocked ERP waveforms for the fastest (20\%) and slowest $(20 \%)$ trials are shown in Figure 4. There, it is obvious that the cue-related P3 is larger for those trials that are responded to faster, proving a direct relationship between preparation and response speed. The statistical significance of this fast-slow difference was tested by a regionof-interest analysis - that is, an ANOVA including only parietal electrodes (P3, Pz, and P4) in the time windows between 200 and $700 \mathrm{msec}$. This analysis revealed main effects of response speed in the intervals between 350 and $400 \mathrm{msec}[F(1,14)=4.13, p=.0615]$ and 450 and $650 \operatorname{msec}[\min F(1,14)=3.97, p=.0661 ; \max F(1,14)=$ $5.52, p=.0340]$.

To conclude, the functional interpretation of the $\mathrm{P} 3$ as a measure of advance preparation in task switching was substantiated by the finding that its amplitude varied with task-switching demands and that it was directly related to task performance.

\section{DISCUSSION}

The present study measured ERPs in a cued taskswitching paradigm with a 2:1 mapping between cues and tasks in order to disambiguate cue switching and task switching on a neurophysiological level. The behavioral data showed evidence for both cue-switch and task-switch costs, replicating the finding that task-switch costs, as measured with the standard task-cuing procedure, are in at least some part due to a change in the task-indicating cue (see Logan \& Bundesen, 2003; Mayr \& Kliegl, 2003).

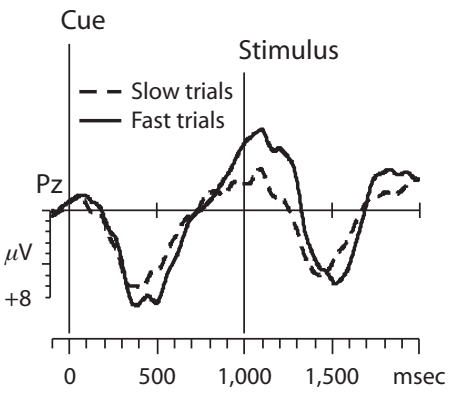

Figure 4. ERPs of the CSI 1,000 condition, averaged according to RTs. Fast trials are accompanied by a larger $\mathbf{P} 3$ amplitude during preparation than are slow trials, indicating that the $\mathbf{P 3}$ amplitude is predictive for subsequent task performance.

Moreover, cue-switch costs were completely eliminated when the CSI increased from 200 to $1,000 \mathrm{msec}$, indicating that performance impairment because of a cue switch can be completely compensated for, provided there is enough time between the presentations of the cue and the stimulus. A reduction with increasing CSI was also observed for the true task-switch costs (see also Monsell \& Mizon, 2006), indicating that participants could and did actually use the cue for advance reconfiguration.

\section{Cue Versus Task Switching}

Aside from the differences in the preparation-related positivity (which will be discussed below), the two CSI conditions showed remarkably similar transition effects. In both CSI conditions, the neural (electrophysiological) correlates of the total switch effect could be split into two components, one associated with a cue switch and the other associated with a task switch. Cue switching already affected the ERPs $300 \mathrm{msec}$ after cue onset and showed a relative negativity, with a centrofrontal maximum, when compared with no-switch trials. Task switching evoked another relative negativity much later in time, starting around $400 \mathrm{msec}$ after stimulus onset, with a topography that significantly differed from that of the cue-switch effect. Accordingly, cue and task switching became manifest in distinct ERP phenomena that differed in both their temporal characteristics and topographical distributions. Therefore, these findings provide evidence that distinct mechanisms underlie cue switching and task switching. Consistent with the assumption that behavioral cueswitch costs arise with the interpretation of the cue, the cue-switch effect in the ERPs is evident during the processing of the cue. This can be observed in the CSI 1,000 condition. However, even with a short interval between cue and stimulus, in which the ERPs of these two events overlapped, the processes associated with cue switching could still be separated from those associated with task switching. The early negative difference thus reflects cuetriggered processes that fully affect RT switch costs in the short-CSI condition but become absorbed when the CSI is long. The late negativity reflects those differences between switch and repetition trials that arise during task execution and that become evident as RT switch costs in both CSI conditions. These distinct features indicate that 
cue- and task-switch costs arise in different processing stages by activation of distinct neural generators.

The centrofrontal focus of the early negative difference here corresponds to the topography of the switch effects during cue processing described in other studies (albeit using different switching procedures; see, e.g., Brass, Ullsperger, Knoesche, von Cramon, \& Phillips, 2005; Miniussi, Marzi, \& Nobre, 2005). In those studies, the frontal effects were attributed to processes associated with the preparation for an actual task switch. In the present study, however, the evidence points to processes related to the encoding or interpretation of the cue, rather than to switching tasks per se. Interestingly, a similar effect was found in a switching study by Gehring and colleagues (Gehring, Bryck, Jonides, Albin, \& Badre, 2003), albeit with a completely different paradigm. In this study, participants observed a stimulus sequence comprising different object stimuli and had to update the appropriate mental count. In the cases in which two consecutive trials required an update of the same count, but with different stimuli, a negative-going wave was observed. This stimulus mismatch effect was found around $300 \mathrm{msec}$, with a frontal maximum, and is therefore very similar to the cueswitch negativity we have observed in the present study. This finding suggests that the cue-switch effect may be related to the relatively automatic detection of a physical mismatch. However, the single-task blocks in our study, in which cue information was not needed to accomplish the task, did not show such an effect. Thus, attentional processing of the cue seems to be a prerequisite for the occurrence of the cue-switch negativity, which therefore seems to be more than a simple mismatch detection process. Rather, it seems to be related to the interpretation of the cue, a process that supposedly is more demanding for cue-change than for cue-repetition trials.

The second component, related to the actual task switch, has a broad distribution similar to those of the stimulus-related effects found in other studies (Karayanidis, Coltheart, Michie, \& Murphy, 2003; Nicholson et al., 2005). In those studies, a switch-related negative difference was interpreted in terms of the demands of counteracting interference from the competing task set. The present study further qualifies this negative difference as a manifestation of true task-switch costs, because it appeared to be unaffected by a mere cue switch.

In other studies, however, similar effects have been interpreted as a modulation of the parietal P3b, with a larger positive amplitude for repetition than for switch trials (see, e.g., Barceló et al., 2002; Hsieh \& Chen, 2006). This interpretation might not be intuitive at first glance, because in terms of the context-updating hypothesis (Donchin \& Coles, 1988), one would expect to find the largest amplitude for the condition that requires the largest amount of working memory revision - that is, the task-switch condition. Such a pattern was observed for the cue-evoked P3, but not for the stimulus-evoked P3. In adherence to the context-updating hypothesis, it has been argued that the P3 attenuation indicates that context updating is impaired or less easily achieved when the context is ambiguous (see Gehring et al., 2003). However, it is also conceivable that the P3 accomplishes different functions during preparation and task execution. This has been already proposed by Barceló et al. (2002), who observed in a variant of the Wisconsin card sorting test a dissociation of P3 effects comparable to the one in the present study: A P3 elicited by feedback stimuli was larger when the feedback indicated a switch to another rule (similar to the task-switch effects in the positivity during preparation in the present study). During card matching, the P3 was larger when the rule stayed the same (similar to the more negative-going potential for task switching during task performance). According to the authors, only the P3 in the feedback stage reflected updating, whereas the one in the matching stage reflected rehearsal of task rules in working memory.

So far, it is difficult to determine which processes are reflected by the stimulus-evoked potentials in our study. We also have to consider that the topography of the present effect is not typical for a P3b modulation: The difference between task switching and repetition is equally pronounced at parietal and frontal electrodes, indicating a mixture of parietal and frontal effects (which might reflect functionally distinct processes), rather than a simple P3b modulation. Further studies are required to address this issue.

Whatever processes are reflected by the stimulusrelated positivity, the data of the present study show that the stimulus-related task-switching effects are functionally dissociable from the mere cue-switch effects arising immediately after cue presentation. We therefore conclude that task-switch costs not only represent more of the same of what was already captured by cue-switch costs, but that task switching involves a unique set of mental processes.

One potential qualification requires attention. In comparison with previous studies using a 2:1 mapping between cues and tasks, the behavioral cue-switch costs in the present study were surprisingly small. This result is even more surprising because the present experiment was closely modeled on those of Mayr and Kliegl (2003), which revealed cue-switch costs that were large relative to task-switch costs but were still smaller than those found by Logan and Bundesen (2003). Practice effects might have influenced the size of the cue-switch costs (see Mayr \& Kliegl [2003, Experiment 1], who found a reduction of cue-switch costs with practice, and Nicholson et al. [2006], who found no cue-switch costs after extensive practice). However, participants in the present study accomplished almost the same number of mixed-block trials as the participants in Experiment 2 of Mayr and Kliegl (2003), in which pronounced cue-switch costs of more than $150 \mathrm{msec}$ were found. Thus, practice effects do not seem to be a plausible explanation for the relatively small cue-switch costs in the present study. The only difference that comes to mind is that, whereas Mayr and Kliegl (2003) used tasks with three response choices each, we instead used four response choices. It is conceivable that task complexity (i.e., the number of choices) determines how strongly a task needs to be implemented, which in turn determines the size of the task-switch cost. In this regard, it is also of interest that Logan and colleagues used two-choice tasks and typically found larger cue-switch and smaller taskswitch costs, relatively speaking, than did Mayr and Klieg1 
(2003). It seems worthwhile to further examine the role of this variable in future work.

Despite the small behavioral effects, the cue-switch contrast in the present study was associated with a distinct and theoretically meaningful physiological signature. Thus, although we may need to be careful in terms of generalizing our results to all task-switching situations, our results do show that it is possible to dissociate cueand task-related processes. It is also of interest that in a very similar design (but with a three-choice task), Bryck, Gordon, and Mayr (2004) found substantial behavioral cue-switch costs, as well as distinct fMRI activation patterns for the cue-switch and task-switch effects.

\section{Cue-Driven Updating}

We proposed that when cues are relevant in principle (i.e., in mixed-task conditions), cue presentation elicits an updating process that can initiate prior to stimulus arrival. Consistent with this expectation, the largest effects in this experiment, both in terms of RTs and ERPs, were found for the contrast between single-task and mixed blocks. In terms of RTs, it is important to note that overall RTs, as well as both cue-switch and task-switch costs, were reduced as a function of CSI, a finding that is broadly consistent with the notion that the long CSI provides the opportunity for updating information in working memory prior to stimulus arrival. In terms of ERPs, this effect appears in the form of a large positivity locked to the cue, and thus occurs before the stimulus in the long-CSI condition, but it could also be observed in the short-CSI condition. The long-CSI condition allowed for an online measurement of these cue-triggered processes, without any confound caused by an overlap with stimulus-related ERPs. In that condition, the cue elicited a positive deflection around 300 to $500 \mathrm{msec}$ with a parietal maximum, with a time course and topography typical for a P3b. Evidence for a functional interpretation of this P3b as a measure of advanced updating during task selection comes from three independent empirical results.

1. The P3 was absent in single-task blocks. With the task remaining constant for a block of 32 trials, the cue would not provide any task-relevant information. This is confirmed by the behavioral data, showing that performance was completely unaffected by the CSI manipulation. There was no evidence for decreasing RTs with longer CSIs, nor any difference between cue-switch and cue-repetition trials. Consistently, a positive deflection was only elicited by cues in the mixed blocks, in which the cues provided relevant information about which task was to be performed on the next stimulus (for similar evidence, see Kray et al., 2005).

2. P3 amplitude was modulated by switching demands. The amplitude was larger for task-switch than for task-repetition trials. This finding is consistent with previous studies that reported that a switch in task set was accompanied by a larger positivity after cue onset, with a parietal maximum (see, e.g., Barceló et al., 2006; Barceló et al., 2002; Karayanidis et al., 2003; Nicholson et al., 2005). Although the labelings of the ERP component and the attributions of underlying mechanisms differ between these studies, they agree on the assumption that the switch-related positivity reflects the activation of an endogenous process-either anticipatory task-set reconfiguration (Karayanidis et al., 2003; Nicholson et al., 2005) or updating of task-set information (see Barceló et al., 2006). The present study supports this interpretation by showing that the differential positivity not only signaled a switch in the task-indicating cue but was an effect that primarily emerged as a difference between taskswitch and task-repetition trials (for similar evidence, see Nicholson et al., 2006).

Our data also show that the processes reflected by the P3 are not specific to task-switch trials. According to Mayr and Kliegl (2000, 2003), an upcoming task can be prepared by retrieving the relevant task rules from longterm memory. In the task-cuing paradigm, this retrieval process seems to be directly related to the task-indicating cue and initiated whenever a cue is presented. Switching demands may affect this process, simply because retrieval is easier in repetition than in switch trials - that is, the relevant information in repetition trials has been retrieved recently and only needs to be refreshed. However, in their original study on cue and task switching, only cue-switch, not task-switch, costs interacted with CSI (Mayr \& Kliegl, 2003), suggesting that attentional reconfiguration needs to wait until the stimulus is presented. In contrast, here we observed a reduction of task-switch costs with increasing time to prepare. Along with ERP evidence for an updating process during the CSI, which appears to have been more demanding when a switch of the task was required, these results suggest that updating and reconfiguration processes, at least in certain situations, can run in advance, before the stimulus is presented (see also Monsell \& Mizon, 2006).

3. The P3 was associated with response speed. The amplitude was larger for those trials that were responded to faster, and therefore reflects trial-by-trial variations in preparation. More efficient updating leads to better performance. An interesting refinement of this finding would be to investigate whether this effect interacts with transition type. From the assumption that advance reconfiguration leads to increased switch costs, one would expect to find larger amplitude differences for fast and slow task-switch than for fast and slow task-repetition trials. The number of trials in the present study was too small to get reliable ERPs for such an analysis. Therefore, further studies should be run to shed more light on this issue.

Combined, these findings provide evidence for task-set updating processes that can run in advance when initiated by a cue. Consistent with the task-retrieval view, every cue seems to elicit a process of retrieving the relevant information from long-term into working memory (see, e.g., Mayr \& Kliegl, 2000, 2003). Even more importantly, the increase in the P3 for switch trials suggests that the updating demands are substantially larger when the task changes than when only cues change.

\section{Conclusions}

All in all, the present results provide neural-level evidence that task sets actually do matter in the cued task-switching paradigm. Although this conclusion may seem obvious, 
the alternative view presented by Logan and colleaguesnamely, that switch costs are due to priming of cue-stimulus compounds (Schneider \& Logan, 2005) - constitutes a serious competitor model. Also, it is possible that critical boundary conditions exist under which task-switch costs disappear - for instance, under a high probability of switch trials (Monsell \& Mizon, 2006). Despite these possibilities, it is important to note that, aside from the present results, other findings have shown that cue-switch and taskswitch components can be dissociated through different experimental manipulations (see, e.g., Mayr, 2006; Mayr $\&$ Kliegl, 2003), and there is also increasing evidence that endogenous task-set reconfiguration processes take place in the interval between cue and stimulus presentation (see Monsell \& Mizon, 2006; Nicholson et al., 2006). Thus, at least in many of the standard cued task-switching situations, task sets seem to be critical representational entities among which selection occurs.

\section{AUTHOR NOTE}

This research was supported by Grant Ro 529/17-2 from the German Research Foundation (DFG) and the Max-Planck award assigned to F.R. We thank Katharina Dobs for programming the stimulus presentation, Lisa Ochs for collecting the data, and Patrick Khader for valuable suggestions and helpful comments. Correspondence may be addressed to K. Jost, Department of Psychology, Philipps University Marburg, Gutenbergstrasse 18, 35032 Marburg, Germany (e-mail: jost@staff .uni-marburg.de).

Note-This article was accepted by the previous editorial team, when John Jonides was Editor.

\section{REFERENCES}

Barceló, F., Escera, C., Corral, M. J., \& Periáñez, J. A. (2006). Task switching and novelty processing activate a common neural network for cognitive control. Journal of Cognitive Neuroscience, 18, 1734-1748.

Barceló, F., Periáñez, J. A., \& Knight, R. T. (2002). Think differently: A brain orienting response to task novelty. NeuroReport, 13, 1887-1892.

Brass, M., Ullsperger, M., Knoesche, T. R., von Cramon, D. Y., \& Phillips, N. A. (2005). Who comes first? The role of the prefrontal and parietal cortex in cognitive control. Journal of Cognitive Neuroscience, 17, 1367-1375.

Bryck, R. L., Gordon, K. B. C., \& MaYr, U. (2004, November). Neuroanatomical correlates of cue switching versus task switching. Poster presented at the 45th Annual Meeting of the Psychonomic Society, Minneapolis.

DE Jong, R. (2000). An intention-activation account of residual switch costs. In S. Monsell \& J. Driver (Eds.), Control of cognitive processes: Attention and performance XVIII (pp. 357-376). Cambridge, MA: MIT Press, Bradford Books.

Donchin, E. (1981). Surprise! . . Surprise? Psychophysiology, 18, 493-513.

Donchin, E., \& Coles, M. G. (1988). Is the P300 component a manifestation of context updating? Behavioral \& Brain Sciences, 11, 357-427.

Gehring, W. J., Bryck, R. L., Jonides, J., Albin, R. L., \& Badre, D. (2003). The mind's eye, looking inward? In search of executive control in internal attention shifting. Psychophysiology, 40, 572-585.

Hennighausen, E., Heil, M., \& Rösler, F. (1993). A correction method for DC drift artifacts. Electroencephalography \& Clinical Neurophysiology, 86, 199-204.
HsieH, S., \& CHEN, P. (2006). Task reconfiguration and carryover in task switching: An event-related potential study. Brain Research, 1084, 132-145.

Huynh, H., \& FELdT, L. S. (1976). Estimation of the Box correction for degrees of freedom from sample data in randomized block and splitplot designs. Journal of Educational Statistics, 1, 69-82.

JASPER, H. (1958). The ten-twenty electrode system of the International Federation. Electroencephalography \& Clinical Neurophysiology, 10, 371-375.

Karayanidis, F., Coltheart, M., Michie, P. T., \& Murphy, K. (2003). Electrophysiological correlates of anticipatory and poststimulus components of task switching. Psychophysiology, 40, 329-348.

Kray, J., Eppinger, B., \& Mecklinger, A. (2005). Age differences in attentional control: An event-related potential approach. Psychophysiology, 42, 407-416.

Logan, G. D., \& Bundesen, C. (2003). Clever homunculus: Is there an endogenous act of control in the explicit task-cuing procedure? Journal of Experimental Psychology: Human Perception \& Performance, 29, 575-599.

Logan, G. D., \& Bundesen, C. (2004). Very clever homunculus: Compound stimulus strategies for the explicit task-cuing procedure. Psychonomic Bulletin \& Review, 11, 832-840.

MAYR, U. (2006). What matters in the cued task-switching paradigm: Tasks or cues? Psychonomic Bulletin \& Review, 13, 794-799.

Mayr, U., \& KLIEGL, R. (2000). Task-set switching and long-term memory retrieval. Journal of Experimental Psychology: Learning, Memory, \& Cognition, 26, 1124-1140.

MAYR, U., \& KLIEgL, R. (2003). Differential effects of cue changes and task changes on task-set selection costs. Journal of Experimental Psychology: Learning, Memory, \& Cognition, 29, 362-372.

McCarThy, G., \& Wood, C. C. (1985). Scalp distributions of event-related potentials: An ambiguity associated with analysis of variance models. Electroencephalography \& Clinical Neurophysiology, 62, 203-208.

Meiran, N. (1996). Reconfiguration of processing mode prior to task performance. Journal of Experimental Psychology: Learning, Memory, \& Cognition, 22, 1423-1442.

Miniussi, C., Marzi, C. A., \& Nobre, A. C. (2005). Modulation of brain activity by selective task sets observed using event-related potentials. Neuropsychologia, 43, 1514-1528.

Monsell, S. (2003). Task switching. Trends in Cognitive Sciences, 7, 134-140.

Monsell, S., \& Mizon, G. A. (2006). Can the task-cuing paradigm measure an endogenous task-set reconfiguration process? Journal of Experimental Psychology: Human Perception \& Performance, 32, 493-516.

Nicholson, R., Karayanidis, F., Bumak, E., Poboka, D., \& Michie, P. T. (2006). ERPs dissociate the effects of switching task sets and task cues. Brain Research, 1095, 107-123.

Nicholson, R., Karayanidis, F., Ровoka, D., Heathcote, A., \& Michie, P. T. (2005). Electrophysiological correlates of anticipatory task-switching processes. Psychophysiology, 42, 540-554.

Rogers, R. D., \& Monsell, S. (1995). Costs of a predictable switch between simple cognitive tasks. Journal of Experimental Psychology: General, 124, 207-231.

Rushworth, M. F. S., Passingham, R. E., \& Nobre, A. C. (2002). Components of switching intentional set. Journal of Cognitive Neuroscience, 14, 1139-1150.

Rushworth, M. F. S., Passingham, R. E., \& Nobre, A. C. (2005). Components of attentional set-switching. Experimental Psychology, 52, 83-98.

Schneider, D. W., \& Logan, G. D. (2005). Modeling task switching without switching tasks: A short-term priming account of explicitly cued performance. Journal of Experimental Psychology: General, 134, 343-367.

(Manuscript received September 6, 2006; revision accepted for publication October 17, 2007.) 